\title{
Pierre Fournier, Travailler dans le nucléaire : enquête au cour d'un site à risques
}

Paris, Armand Colin, coll. « Sociétales », 2012

\section{Gabrielle Hecht}

\section{OpenEdition}

\section{Journals}

Édition électronique

URL : https://journals.openedition.org/travailemploi/6167

DOI : 10.4000/travailemploi.6167

ISSN : 1775-416X

Éditeur

DARES - Ministère du Travail

Édition imprimée

Date de publication : 14 décembre 2013

Pagination : 86-88

ISSN : 0224-4365

\section{Référence électronique}

Gabrielle Hecht, «Pierre Fournier, Travailler dans le nucléaire : enquête au cœur d'un site à risques » Travail et Emploi [En ligne], 136 | octobre-décembre 2013, mis en ligne le 01 octobre 2013, consulté le

30 juillet 2021. URL : http://journals.openedition.org/travailemploi/6167 ; DOI : https://doi.org/

10.4000/travailemploi.6167

Ce document a été généré automatiquement le 30 juillet 2021.

(C) Direction de l'animation de la recherche, des études et des statistiques (Dares) 


\title{
Pierre Fournier, Travailler dans le nucléaire : enquête au cœur d'un site à risques
}

Paris, Armand Colin, coll. « Sociétales », 2012

\author{
Gabrielle Hecht
}

\section{RÉFÉRENCE}

Pierre Fournier, Travailler dans le nucléaire : enquête au cœur d'un site à risques, Paris, Armand Colin, coll. « Sociétales », 2012, 231 p.

1 Ce livre présente une enquête tout à fait remarquable sur le travail dans les sites nucléaires à travers une réflexion qui se situe au croisement de la sociologie, de l'ethnographie et du témoignage. En effet, le sociologue Pierre Fournier est fils d'un employé du nucléaire. Embauché comme stagiaire dans une entreprise de soustraitance sur le même site que son père, il est rentré très jeune au cœur du travail nucléaire. S'ensuivent un parcours universitaire, et de nombreux travaux de recherche sur le monde du travail en milieu rural et urbain... pour aboutir enfin à cette enquête menée sur le site même où il avait été stagiaire deux décennies auparavant. Ce parcours est important pour saisir la singularité du livre: il s'agit de la seule ethnographie vraiment participative qui traite du travail nucléaire. D’autres chercheurs en sciences sociales ont certes observé le travail nucléaire, interviewé les ouvriers ou même pris part à des réunions. Mais Fournier est le seul à avoir réellement travaillé pendant des mois dans un milieu radioactif, au même titre que des ouvriers "normaux ». Cette participation a de surcroît inspiré confiance au service des ressources humaines qui accorde à Fournier l'accès inédit à plusieurs centaines de dossiers de personnels actifs, qu'il dépouille (en respectant bien l'anonymat) afin d'approfondir l'enquête.

2 L'analyse qui en découle nous propose un regard bien plus nuancé et mesuré que d'ordinaire sur le travail en milieu nucléaire. Trop souvent, en effet, l'étude de ce 
dernier s'attache uniquement aux dangers invisibles ou mal appréciés, aux normes non respectées; on peut en ressortir avec l'impression que l'activité ne consiste en rien d'autre qu'en un effort considérable de la part des entreprises pour contourner la réglementation et de déni psychologique de la part des ouvriers. Or, ici, Fournier rend à l'activité tout son caractère industriel. À travers une analyse approfondie de quelques interventions, on comprend que - comme n'importe quel travail dans n'importe quelle industrie complexe - le travail nucléaire nécessite un apprentissage poussé, des compétences fines en matière d'instrumentation, des négociations compliquées autour des savoirs de chacun. En même temps, on ne peut jamais laisser de côté le fait que tout se déroule en milieu radioactif, ce qui conduit à des mesures tout à fait exceptionnelles. Ainsi, nous suivons une équipe lors d'une " plongée », c'est-à-dire une intervention de maintenance dans une zone hautement radioactive, qui nécessite des tenues et des équipements très spécifiques. Fournier nous fait découvrir le moindre détail: la manière de mettre la cagoule et de scotcher les gants ; l'entrée en zone contrôlée ; la difficulté à se mouvoir et la sensation d'étouffement que l'on éprouve dans une combinaison quasi lunaire; la sérénité des uns et la panique des autres; les "gauloiseries" échangées après l'intervention, redoublées par la présence d'une femme, la seule, au sein de l'équipe. On aurait d'ailleurs apprécié une analyse plus fine et plus poussée des dynamiques de genre dans ce milieu de travail très masculin. Homme ou femme, la sécurité de l'un y dépend des gestes et des compétences de l'autre: ainsi, les ouvriers forment "un collectif serré ", même si les différences de statut, d'ancienneté, ou de rang ont toujours leur importance dans le déroulement de l'opération. Ces descriptions denses nous donnent à voir les ouvriers du nucléaire naviguant constamment à la frontière entre banalité et singularité.

3 Le thème de l'erreur - qui domine d'ailleurs toutes les discussions sur la sûreté nucléaire, que ce soit au sein du secteur ou dans le débat public - traverse le livre. Comment peut-on expliquer un accident quel qu'il soit - qu'il s'agisse d'un accident comme Fukushima ou le moment où un décontaminateur chargé de l'emballage des déchets renverse un pot de substances radioactives sur sa jambe ? Afin d'analyser ce dernier incident, Fournier reconstruit l'enchaînement d'actions qui y aboutit. Ainsi, on découvre que le pot en question n'était pas étanche en arrivant chez le décontaminateur, comme il aurait dû l'être; le tuyauteur qui aurait dû le sceller ne l'avait pas fait correctement parce qu'il était pressé ; ou plutôt, le tuyauteur était inquiet, car le liquide issu de la canalisation qu'il venait de vidanger avait rempli trois pots ; or, il savait que pour éviter un accident critique, il ne fallait pas garder plus d'un pot dans la salle; si autant de produit était sorti de la canalisation, c'était sans doute parce qu'elle avait mal été vidangée ; par la suite, le décontaminateur accidenté ne s'est pas vu prodiguer les soins adéquats par l'agent de radioprotection; etc. On découvre donc qu'un accident que l'on serait tenté d'attribuer à « l'erreur humaine » est en fait distribué tout au long d'une chaîne composée d'humains et de non-humains (robinets, normes, pots, etc.), de sorte qu'il est impossible d'établir une échelle stricte des causalités et de déterminer, parmi les causes d'erreurs possibles, lesquelles sont d'ordre individuel, institutionnel voire national. Entendons-nous bien, Fournier ne déploie nullement ce vocabulaire latourien... ce que l'on peut regretter, car une utilisation judicieuse l'aurait aidé à approfondir son analyse. Finalement, « l'erreur humaine » - ce « fétiche » brandi par l'industrie nucléaire pour expliquer tout manquement - explique en fait très peu. 
4 Mais Fournier s'en prend aussi à une autre explication "fétiche», celle qui laisse entendre que c'est "la pression économique " qui conduit forcément à ce que les opérations de maintenance soient effectuées rapidement, et que les réglementations et procédures soient négligées. Sans nier la réalité d'une telle pression, Fournier montre qu'elle ne détermine pas automatiquement le déroulement des opérations au jour le jour. S'appuyant sur un long entretien avec un ancien employé à la retraite qui, après avoir commencé sa carrière comme technicien de maintenance devint agent de maitrise puis cadre, l'auteur insiste sur le fait que les ouvriers ne sont «pas vraiment des kamikazes »; les voir ainsi revient à nier la complexité d'une situation dans laquelle les questions budgétaires occupent certes une place importante mais interagissent avec d'autres enjeux.

5 Le lecteur pourra découvrir bien d'autres aspects passionnants dans ce livre riche et captivant, tels que l'évolution du métier de radioprotectionniste, ou les enjeux liés au renouvellement des générations d'ouvriers sur le site ainsi qu'à la cohabitation entre générations; ou bien encore, les impacts subtils sur le travail quotidien de la bataille entre industriels et écologistes autour du nucléaire. Il pourra aussi regretter certaines absences, qui sont d'ailleurs peut-être dues à des contraintes imposées par l'éditeur : un manque de réflexivité autour de la démarche méthodologique, de sorte que l'on n'est pas toujours sûr de la position de l'auteur lors d'une intervention (était-il présent en tant que stagiaire ou chercheur? Est-ce qu'il a pris des notes lors de son stage dans les années 1990 ou s'appuie-t-il entièrement sur sa mémoire ?) ; une réflexion théorique un peu légère vis-à-vis d'une part, de la littérature sur le nucléaire et, d'autre part, de celle de la sociologie des techniques et des risques; l'absence presque totale de la littérature anglo-saxonne sur le sujet (en particulier de la magnifique ethnographie de Constance Perin $^{1}$ sur la manière dont le risque radioactif est appréhendé par les ouvriers américains). Mais ce ne sont finalement que des petits bémols dans un livre passionnant et unique, qui vaut bien une lecture approfondie.

\section{NOTES}

1. Perin C. (2005), Shouldering risks: the culture of control in the nuclear power industry, Princeton (N.

J.), Princeton university press.

\section{AUTEURS}

\section{GABRIELLE HECHT}

Université du Michigan 